\title{
Atypical Takotsubo Cardiomyopathy With Persistent Left Ventricular Aneurysm After Cardiac Surgery
}

\author{
Xiaojie Zhang \\ Zhejiang University School of Medicine, Sir Run Run Shaw Hospital, Zhejiang, China
}

\section{ABSTRACT}

A 57-year-old man was admitted to the hospital because of severe mitral regurgitation. Minimally invasive mitral valvuloplasty was performed. Six months after surgery, he presented with discomfort. Urgent coronary angiography showed a normal coronary artery and large left ventricular aneurysm. After atypical Takotsubo cardiomyopathy was diagnosed, his clinical symptoms and heart function were improved with medication for his persistent left ventricular aneurysm. After 24 hours, Hotler showed a lethal ventricular arrhythmia. Surgical treatment was recommended and refused. After four years observation, he died of asystole. Takotsubo cardiomyopathy is considered a severe complication after surgery. The prognosis is very poor if it has catastrophic complications.

\section{INTRODUCTION}

Typical Takotsubo cardiomyopathy is a cardiac syndrome characterized by reversible left ventricular dysfunction and left ventricular apical ballooning deformity with clinical symptoms and signs similar to acute coronary syndrome but with a negative coronary angiography (CAG). It predominantly has been observed in woman older than 60 years of age, often is triggered by emotional or physical stress, and associated with sympathetic stimulation mediated by excessive plasma catecholamine levels [Kudaiberdiev 2017]. Generally, patients with Takotsubo cardiomyopathy have a favorable prognosis because the wall motion abnormality returns to normal within several days to weeks without severe complication. Here, we describe an atypical Takotsubo cardiomyopathy with a lethal complication six months after cardiac surgery.

\section{CASE REPORT}

A 57-year-old man was admitted to the hospital two months after stroke. Transthoracic echocardiography (TTE) confirmed severe mitral regurgitation and electrocardiogram

Received February 18, 2021; received in revised form March 19, 2021; accepted March 22, 2021.

Correspondence: Xiaojie Zhang, Zhejiang University School of Medicine, Sir Run Run Shaw Hospital, Zhejiang, China (e-mail:drzhangxiaojie@zju.edu.cn).
(ECG) showed no other associated alterations. The CAG was negative. Other routine preoperative assessment did not reveal remarkable findings.

Minimally invasive mitral valvuloplasty was performed from a right fourth intercostal space. The intraoperative TEE showed normal prosthesis positioning and function and no obvious wall motion abnormalities of the left ventricle. $\mathrm{CPB}$ time was 77 minutes and aortic cross-clamp time was 58 minutes. He was transferred to the intensive care unit (ICU), extubated seven hours later, and returned for ordinary ward a day later. Norepinephrine and milrinone routinely were prescribed in ICU. After six days, the patient was smoothly discharged.

One month after surgery, the patient felt no discomfort, while TTE showed the diffusely weakened motion of the left ventricular postwall (EF: 36\%). It was the same TTE result three months after surgery. However, TTE showed the left ventricle dilated, segmental abnormal activities of the left ventricular postwall (EF: 27.4\%), and ventricular aneurysm located in the basal and middle part of the left ventricular postwall six months after surgery. The size was $45.6^{*} 17.3 \mathrm{~mm}$. While the hemodynamic was stable, he presented with chest tightness and dizziness. The ECG indicated the ST-T alteration in the anterolateral and inferior wall of left ventricle. The urgent CAG was performed and negative, as was the result of CK-MB and Troponin. The medication treatment with beta-blockers and loop diuretics had been taken after operation. After a month, TTE showed the EF was elevated to $45 \%$, despite the ventricular aneurysm remaining unchanged. Twenty-four-hour Hotler showed sinus rhythm with frequent ventricular premature beats and paroxysmal ventricular tachycardia, while the ECG indicated T-wave inversion in the inferior wall. Amiodarone was not effective. Surgical treatment, including biventricular pacemaker and ICD implantation, was recommended and refused for financial reasons. Despite medication, he still occasionally complained of chest tightness and dizziness during follow up. After four years observation, the patient was taken by ambulance to the hospital, where CPR failed for 40 minutes because of sudden cardiac arrest at home (Figure 1 and 2).

\section{DISCUSSION}

Previous cases have been reported that atypical forms in a minority, including $14.6 \%$ of patients present a midventricular distension, $2.2 \%$ are characterized by a basal type 


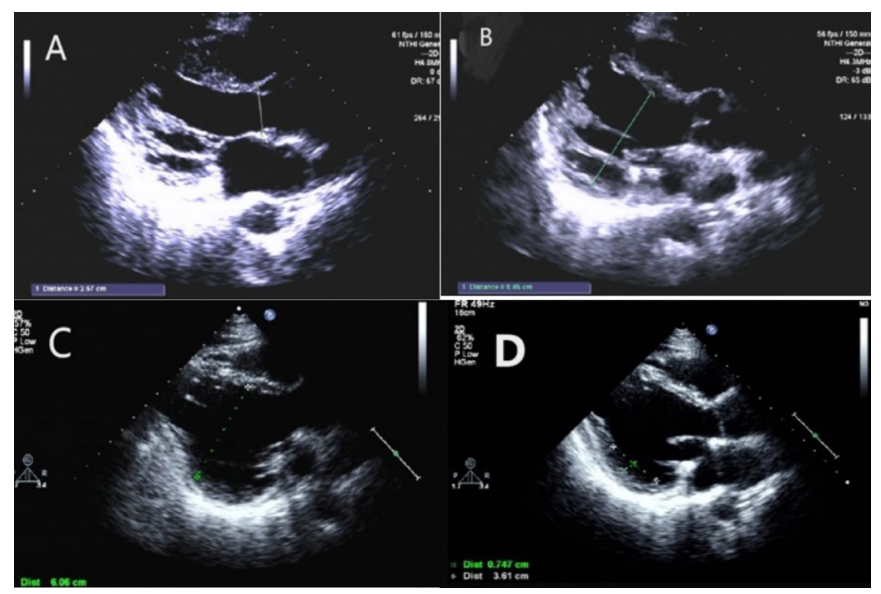

Figure 1. A, Preoperative echocardiography; B, Postoperative echocardiography (1 month); C and D, Postoperative echocardiography (6 months).

deformity, and $1.5 \%$ present focal areas of ventricular distension and transient hypokinesia [Chiariello 2016]. The primary cause remains undetermined. Analyzing 1750 cases, Templin et al reported that Takotsubo cardiomyopathy was triggered more frequently by a physical rather than emotional stress [Templin 2015]. In the literature, Takotsubo cardiomyopathy after surgery has been reported [Rodrigues 2015]. In 2007, Itoh et al first described the case of a 59-year-old female operated on for mitral valve repair who during the postoperative course showed an elevated ST-segment on electrocardiogram with left ventricle apical ballooning. It was related to regional myocardial stunning caused by cardiopulmonary bypass [Itoh 2007]. Next, Wong et al also reported the case of a 56-yearold female operated on for a large left atrial myxoma on the first postoperative day. The patients complained of sudden chest pain. Electrocardiogram showed new onset ST elevation and T-wave inversion. Cardiac enzymes were mildly elevated. Echocardiogram and left ventriculography showed impaired left ventricular function with apical ballooning deformity. Coronary arteries were normal. When adrenaline infusion was stopped, enzyme and electrocardiogram normalized and left ventricular function improved [Wong 2008]. At present, it is believed that the incidence of Takotsubo cardiomyopathy after cardiac surgery can be reduced by shortening the cardiac arrest time, paying close attention to perioperative myocardial protection, and relieving myocardial ischemiareperfusion injury and coronary spasm.

Most reports of death occurring in Takotsubo cardiomyopathy patients show cardiac wall rupture with hemopericardium or thromboembolic complications, whereas cardiogenic shock, severe heart failure and malignant arrhythmias rarely have been reported [Del Buono 2017]. Our patient was a male with sudden cardiac arrest of atypical Takotsubo cardiomyopathy with persisitent left ventricular inferior aneurysm after minimally invasive mitral valvuloplasty. So far, it has not previously been shown in patients following cardiac surgery. Medication is considered to be the first choice in the treatment of Takotsubo cardiomyopathy. Diuretics

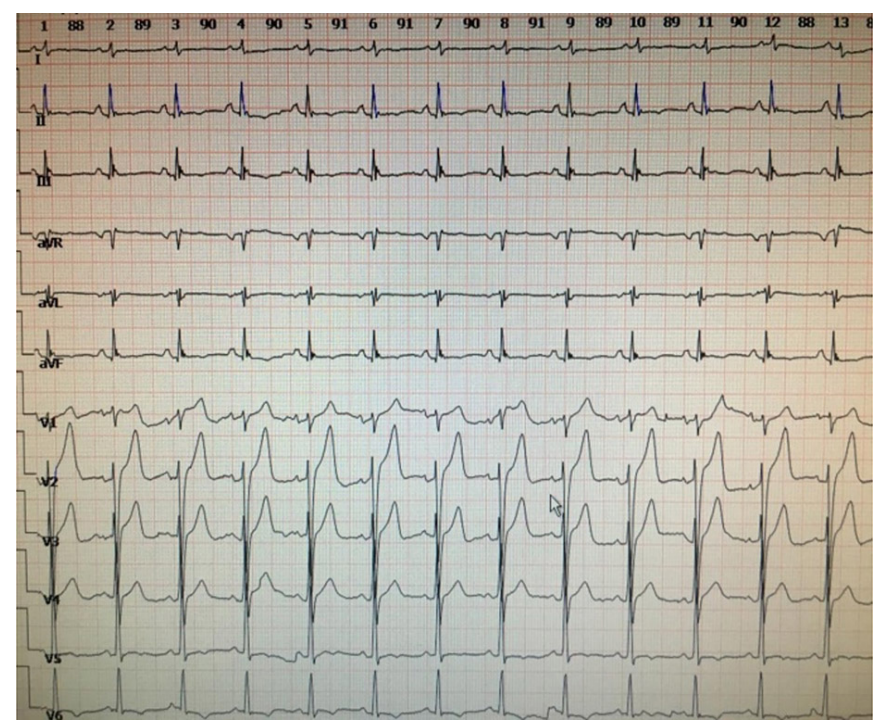

Figure 2. Electrocardiogram during Takotsubo syndrome.

and beta-blockers commonly are used for the treatment of Takotsubo cardiomyopathy. Other treatment agents that can be used include angiotensin converting enzyme inhibitors, angiotensin II type 1 receptor blockers, aldosterone antagonist and anxiolytics. Generally, heart failure is reversible and prognosis may be favorable if appropriate conservative medical treatment promptly is started. In our case, although heart dysfunction was significantly improved with Metoprolol and furosemide, the left ventricular aneurysm remained unchanged and 24-hour Hotler also showed frequent ventricular premature beats and paroxysmal ventricular tachycardia. In a recent review of the literature, Syed et al found in Takotsubo cardiomyopathy patients a $2.2 \%$ prevalence of ventricular fibrillation, $1.2 \%$ of sustained ventricular tachycardia, $1 \%$ of nonsustained ventricular tachycardia, $0.5 \%$ of asystole, $2.9 \%$ of atrioventricular block, $1.3 \%$ of sinus node dysfunction, $4.7 \%$ of atrial fibrillation, and $1.1 \%$ of sudden cardiac death [Syed 2011]. Therefore, it also is considered to be a clinical condition at risk for lethal arrhythmias. It has been reported to show an early mortality of 3-5\% [Rodrigues 2015; Lyon 2016]. Surgical intervention should be considered in the presence of hemodynamic instability, refractory heart failure or ventricular tachyarrhythmias, especially in a subpopulation with QTc $>500 \mathrm{msec}$ in acute phase and/or with right ventricular involvement [Syed 2011]. Miyata et al performed surgical left ventriculoplasty to restore the dilated and distorted left ventricular cavity when left ventricular function did not improve and further worsened, evolving into a large left ventricular aneurysm six months later after medication. The prognosis is very satisfactory [Miyata 2013]. Jesel et al showed life-threatening arrhythmias occur early in patients presenting with TTC and are associated with significantly worse short- and long-term prognosis. For such patients, it is necessary to recommend device therapy, such as biventricular and ICD implantation, because it improves the prognosis [Jesel 2018]. 


\section{CONCLUSION}

Although patients with Takotsubo cardiomyopathy have a favorable prognosis, it is considered a severe complication after heart surgery. We must be aware that the prognosis is very poor if it has catastrophic complications, such as persistent left ventricular aneurysm and ventricular arrhythmia.

\section{REFERENCES}

Chiariello GA, Bruno P, Colizzi C, Crea F, Massetti M. 2016. Takotsubo Cardiomyopathy Following Cardiac Surgery. J Card Surg. Feb;31(2):89-95.

Del Buono MG, O'Quinn MP, Garcia P, et al. 2017. Cardiac arrest due to ventricular fibrillation in a 23 -year old woman with broken heart syndrome. Cardiovasc Pathol. 30:78-81.

Itoh H, Miyake Y, Hioki I, et al. 2007. Report of Takotsubo cardiomyopathy occurring during cardiopulmonary by- pass. J Extra Corpor Technol. 39:109-111.

Jesel L, Berthon C, Messas N, Lim HS, Girardey M, Marzak H, Marchandot B, Trinh A, Ohlmann P, Morel O. 2018. Ventricular arrhythmias and sudden cardiac arrest in Takotsubo cardiomyopathy: Incidence, predictive factors, and clinical implications. Heart Rhythm. 15(8):1171-1178.

Kudaiberdiev T, Akhmedova I, Imanalieva G, Abdildaev I, Jooshev K, Ashimov J, Mirzabekov A, Gaybildaev J. 2017. Surgical treatment of left ventricular wall rupture, regarded as a consequence of Takotsubo cardiomyopathy. SAGE Open Med Case Rep. Jan 18;5:2050313X16689210.

Lyon AR, Bossone E, Schneider B, et al. 2016. Current state of knowledge on Takotsubo syndrome: a position statement from the taskforce on Takotsubo syndrome of the Heart Failure Association of the European Society of Cardiology. Eur J Heart Fail. 18:8-27.

Miyata M, Nakazato K, Sakamoto N, et al. 2013. Left ventricular plasty improved cardiac function in a case of takotsubo cardiomyopathy with persistent aneurysm. J Cardiol Cases. 7:133-136.

Rodrigues LB, Batista A, Monteiro F, et al. 2015. ST-segment elevation during general anesthesia for non-cardiac surgery: A case of takotsubo. Rev Bras Anestesiol. 65:403-406.

Syed FF, Asirvatham SJ, Francis J. 2011. Arrhythmia occurrence with takotsubo cardiomyopathy: A literature review. Europace. 13:780-788.

Templin C, Ghadri JR, Diekmann J, et al. 2015. Clinical features and outcomes of takotsubo (stress) cardiomyopathy. N Engl J Med. 373:929-938.

Wong CP, Jim MH, Chau EM, et al. 2008. Iatrogenic Tako-Tsubo cardiomyopathy. Int J Cardiol. 125:16-18. 